\title{
Effects of the core radius of an isothermal ellipsoidal gravitational lens on the caustics and the critical curves
}

\author{
H. Asada ${ }^{1,2}$ \\ ${ }^{1}$ GReCO, Institute for Astrophysics at Paris, 98bis boulevard Arago, 75014 Paris, France \\ e-mail: asada@phys.hirosaki-u.ac.jp \\ ${ }^{2}$ Faculty of Science and Technology, Hirosaki University, Hirosaki 036-8561, Japan
}

Received 3 March 2004 / Accepted 28 July 2004

\begin{abstract}
We study the effect of the core radius of an isothermal ellipsoidal gravitational lens on the caustics and the critical curves. We derive an analytic expression of the caustics for an isothermal ellipsoidal gravitational lens via a sixth-order algebraic equation. Since the expression is too long, by using another method we obtain a parametric representation of the critical curves in order to show analytically that there exist three cases: there are two curves for a small core radius, one for a quite large one, and no curves appear for an extremely large one, though the latter two cases are not realistic. The caustics are represented also by the same parameter.
\end{abstract}

Key words. gravitational lensing - galaxies: general - cosmology: theory

\section{Introduction}

Gravitational lensing due to a galaxy is indispensable to probe its mass distribution and determine cosmological parameters (for reviews, Schneider et al. 1992). Cored isothermal ellipsoids are often used for modeling a galactic lens. Although such a model is quite simple, it enables us to understand many physical properties of the galactic lens. In addition, it fits with mass profiles implied by observations (e.g., Binney \& Tremaine 1987).

The caustics are curves on the source plane perpendicular to the line of sight. They are important to understand the gravitational lensing, particularly when estimating its probability. Furthermore, the number of images changes if a source crosses the caustics. Similarly, critical curves located on the lens plane play a crucial role; giant arcs are observed near these critical curves.

Analytic expressions for the caustics and critical curves were found for a singular isothermal ellipsoidal lens (Asada et al. 2003) during an algebraic study of the gravitational lens (Asada 2002; Asada et al. 2002). However, a cored isothermal ellipsoidal lens, which is more important and realistic than a singular one, seemed beyond an analytic approach, because the equation for a cored isothermal ellipsoidal lens is of much higher order and thus more complicated from an algebraic point of view (Asada et al. 2003). The existence of a core makes a crucial difference even if the core is small. For instance, the number of images for a singular isothermal ellipsoid is two or four, while it is one, three or five for a cored isothermal one (Asada et al. 2003). The main purpose of the present paper is to study the effect of the core radius on the caustics and the critical curves.

This paper is organized as follows. In Sect. 2, the lens equation for a cored isothermal ellipsoid is given in the form of a single sixth-order algebraic equation. The discriminant for this algebraic equation is also discussed. A parametric representation of the critical curves and caustics are obtained in Sect. 3. Section 4 provides our conclusion.

\section{Lens equation for an isothermal ellipsoidal lens}

We consider an isothermal ellipsoidal lens with ellipticity $0 \leq$ $\epsilon<1 / 5$ and the angular core radius $c$. The surface mass density projected onto the lens plane must be non-negative everywhere. This puts a constraint as $\epsilon<1$. A tighter constraint $\epsilon<1 / 5$ comes from the requirement that the density contour must be convex, which is reasonable for an isolated relaxed system. The lens equation is expressed as

$$
\begin{aligned}
& \beta_{1}=\theta_{1}-\frac{(1-\epsilon) \theta_{1}}{\sqrt{(1-\epsilon) \theta_{1}^{2}+(1+\epsilon) \theta_{2}^{2}+c^{2}}} \\
& \beta_{2}=\theta_{2}-\frac{(1+\epsilon) \theta_{2}}{\sqrt{(1-\epsilon) \theta_{1}^{2}+(1+\epsilon) \theta_{2}^{2}+c^{2}}}
\end{aligned}
$$

where $\boldsymbol{\beta}=\left(\beta_{1}, \beta_{2}\right)$ and $\boldsymbol{\theta}=\left(\theta_{1}, \theta_{2}\right)$ denote the positions of the source and images, respectively. 
For simplicity, we introduce variables as $x \equiv \sqrt{1-\epsilon} \theta_{1}$, $y \equiv \sqrt{1+\epsilon} \theta_{2}, a \equiv \sqrt{1-\epsilon} \beta_{1}$ and $b \equiv \sqrt{1+\epsilon} \beta_{2}$, so that the lens equation can be rewritten as

$a=x\left(1-\frac{1-\epsilon}{\sqrt{x^{2}+y^{2}+c^{2}}}\right)$,

$b=y\left(1-\frac{1+\epsilon}{\sqrt{x^{2}+y^{2}+c^{2}}}\right)$.

For off-axis sources, this lens equation is reduced to a single sixth-order algebraic one as (Asada et al. 2003)

$$
\begin{aligned}
& (a-x)^{2}\left[\left(x^{2}+c^{2}\right)[(1+\epsilon) a-2 \epsilon x]^{2}+(1-\epsilon)^{2} b^{2} x^{2}\right] \\
& \quad-(1-\epsilon)^{2} x^{2}[(1+\epsilon) a-2 \epsilon x]^{2} \\
& \quad=0 .
\end{aligned}
$$

The other component is uniquely determined by

$y=\frac{(1-\epsilon) b x}{(1+\epsilon) a-2 \epsilon x}$.

It should be noted that the positions of the images can be found analytically for a source on a symmetry axis (Asada et al. 2003). However, it is sufficient to consider a case of offaxis sources to obtain expressions of the caustics and critical curves because these expressions are continuous functions of the source position.

By the discriminant for polynomial equations, we can determine a location where the number of real solutions changes (e.g., van der Waerden 1966), which corresponds to a change in the number of images in the context of the lensing. The discriminant for the sixth-order Eq. (5) is computed for instance by using the software Mathematica (Wolfram 2000). The result is a lengthy polynomial of 656 terms, which is not tractable. Hence we employ another method to obtain a parametric representation of the caustics and critical curves in a much shorter and thus more practical form.

\section{Critical curves and caustics}

The critical curves are expressed as the vanishing of the Jacobian of the lens mapping, namely $\partial(a, b) / \partial(x, y)=0$. It becomes

$$
\begin{gathered}
\left(x^{2}+y^{2}+c^{2}\right)^{2}-\left(x^{2}+y^{2}+2 c^{2}+e\left(x^{2}-y^{2}\right)\right) \\
\times\left(x^{2}+y^{2}+c^{2}\right)^{1 / 2}+c^{2}\left(1-e^{2}\right)=0 .
\end{gathered}
$$

This is rewritten as

$$
\begin{gathered}
\left(R+c^{2}\right)^{2}-\left(R+2 c^{2}\right)\left(R+c^{2}\right)^{1 / 2}+c^{2}\left(1-e^{2}\right) \\
=e R\left(R+c^{2}\right)^{1 / 2} \cos 2 \phi,
\end{gathered}
$$

where we introduced the polar coordinates $(x, y)=$ $(r \cos \phi, r \sin \phi)$ and defined $R=r^{2}$.

We will find allowed regions among $r, c$ and $e$, which is due to $-1 \leq \cos 2 \phi \leq 1$ in Eq. (8). In short, Eq. (8) implies

$$
\begin{aligned}
& \left(R+c^{2}\right)^{2}-\left(R+2 c^{2}\right)\left(R+c^{2}\right)^{1 / 2}+c^{2}\left(1-e^{2}\right) \\
& \quad \leq e R\left(R+c^{2}\right)^{1 / 2}
\end{aligned}
$$

and

$$
\begin{gathered}
\left(R+c^{2}\right)^{2}-\left(R+2 c^{2}\right)\left(R+c^{2}\right)^{1 / 2}+c^{2}\left(1-e^{2}\right) \\
\geq-e R\left(R+c^{2}\right)^{1 / 2} .
\end{gathered}
$$

First, we consider Eq. (9), which is rewritten as

$$
\begin{aligned}
(R+ & \left.c^{2}\right)^{2}+c^{2}\left(1-e^{2}\right) \\
& \geq\left(R(1-e)+2 c^{2}\right)\left(R+c^{2}\right)^{1 / 2} .
\end{aligned}
$$

Since the right-hand side of this inequality is positive for $e<1$, this is recast in a polynomial form so that we can find the allowed region for $R$ in an algebraic manner. This polynomial expression is

$$
\begin{aligned}
F(R) \equiv & \left(\left(R+c^{2}\right)^{2}+c^{2}\left(1-e^{2}\right)\right)^{2} \\
& -\left(R(1-e)+2 c^{2}\right)^{2}\left(R+c^{2}\right) \\
\geq & 0 .
\end{aligned}
$$

The discriminant $D_{4}$ of the fourth-order polynomial $F(R)$ is obtained (e.g. van der Waerden 1966) as

$$
\begin{aligned}
D_{4}= & -27 c^{8}(1+e)^{4} \\
& \times\left(\left(1-3 e+3 e^{2}-e^{3}\right)^{2}-c^{4}(1+e)^{2}\right)^{2} .
\end{aligned}
$$

This is non-positive, so that $F(R)=0$ has two real roots as $R=(1-e)^{2}-c^{2}$ and $c^{4 / 3}(1+e)^{2 / 3}-c^{2}$. We must find the smaller root satisfying a non-negativity of $R$. In short, we compare between $0,(1-e)^{2}-c^{2}$ and $c^{4 / 3}(1+e)^{2 / 3}-c^{2}$. There exist four cases for Eq. (12), depending on $c$ as

$$
\begin{aligned}
& \text { for } \quad c<\frac{(1-e)^{3 / 2}}{(1+e)^{1 / 2}} \\
& 0 \leq R \leq c^{4 / 3}(1+e)^{2 / 3}-c^{2}, \\
&(1-e)^{2}-c^{2} \leq R, \\
& \text { for } \frac{(1-e)^{3 / 2}}{(1+e)^{1 / 2}}<c<1-e, \\
& 0 \leq R \leq(1-e)^{2}-c^{2}, \\
& c^{4 / 3}(1+e)^{2 / 3}-c^{2} \leq R,
\end{aligned}
$$

Next, we consider Eq. (10), which implies

$$
\begin{aligned}
G(R) \equiv & \left(\left(R+c^{2}\right)^{2}+c^{2}\left(1-e^{2}\right)\right)^{2} \\
& -\left(R(1+e)+2 c^{2}\right)^{2}\left(R+c^{2}\right) \\
\leq & 0,
\end{aligned}
$$


whose discriminant $D_{4}^{\prime}$ is obtained (e.g. van der Waerden 1966) as

$$
\begin{aligned}
D_{4}^{\prime}= & -27 c^{8}(1-e)^{4} \\
& \times\left(\left(1+3 e+3 e^{2}+e^{3}\right)^{2}-c^{4}(1-e)^{2}\right)^{2} .
\end{aligned}
$$

This is non-positive, so that $G(R)=0$ has two real roots as $R=(1+e)^{2}-c^{2}$ and $c^{4 / 3}|1-e|^{2 / 3}-c^{2}$. We compare between 0 , $(1+e)^{2}-c^{2}$ and $c^{4 / 3}|1-e|^{2 / 3}-c^{2}$. We find three cases for Eq. (20) as

for $c<1-e$,

$$
c^{4 / 3}|1-e|^{2 / 3}-c^{2} \leq R \leq(1+e)^{2}-c^{2},
$$

for $1-e<c<1+e$,

$$
0 \leq R \leq(1+e)^{2}-c^{2},
$$

for $1+e<c$,

no allowed region for $R$.

Bringing together Eqs. (14)-(19) and Eqs. (22)-(24), we find four cases for Eqs. (9) and (10):

$$
\begin{aligned}
& \text { For } c<\frac{(1-e)^{3 / 2}}{(1+e)^{1 / 2}} \text {, } \\
& c^{4 / 3}|1-e|^{2 / 3}-c^{2} \leq R \leq c^{4 / 3}(1+e)^{2 / 3}-c^{2}, \\
& (1-e)^{2}-c^{2} \leq R \leq(1+e)^{2}-c^{2}, \\
& \text { for } \frac{(1-e)^{3 / 2}}{(1+e)^{1 / 2}}<c<1-e \text {, } \\
& c^{4 / 3}|1-e|^{2 / 3}-c^{2} \leq R \leq(1-e)^{2}-c^{2}, \\
& c^{4 / 3}(1+e)^{2 / 3}-c^{2} \leq R \leq(1+e)^{2}-c^{2},
\end{aligned}
$$

for $1-e<c<1+e$,

$$
c^{4 / 3}(1+e)^{2 / 3}-c^{2} \leq R \leq(1+e)^{2}-c^{2},
$$

for $1+e<c$,

$$
\text { no allowed region for } R \text {. }
$$

These cases show that there are two critical curves for a small core radius $c<1-e$, one for the quite large case $1-e<c<$ $1+e$, and they disappear for an extremely large one, $1+e<c$. This anomalous behavior where the critical curves disappear is physically understood as follows. As the core radius becomes much larger, the lens system approaches an approximately constant mass sheet. Around $c \sim 1+e$, the size of the core is comparable to the Einstein ring radius. As a result, such a lens system cannot produce multiple images, which means that there are no critical curves. Furthermore, the latter two cases of large cores are not realistic. Indeed, $c \sim 1$ implies that the core radius is comparable to the Einstein ring radius, which becomes about $10 \mathrm{kpc}$ for a galactic lens with $10^{12}$ solar mass at a high redshift. The core radius of a galaxy is usually much smaller than $10 \mathrm{kpc}$.

It is convenient to consider Eq. (8) as an $r$-parameter representation of the critical curves, because we must solve Eq. (8)

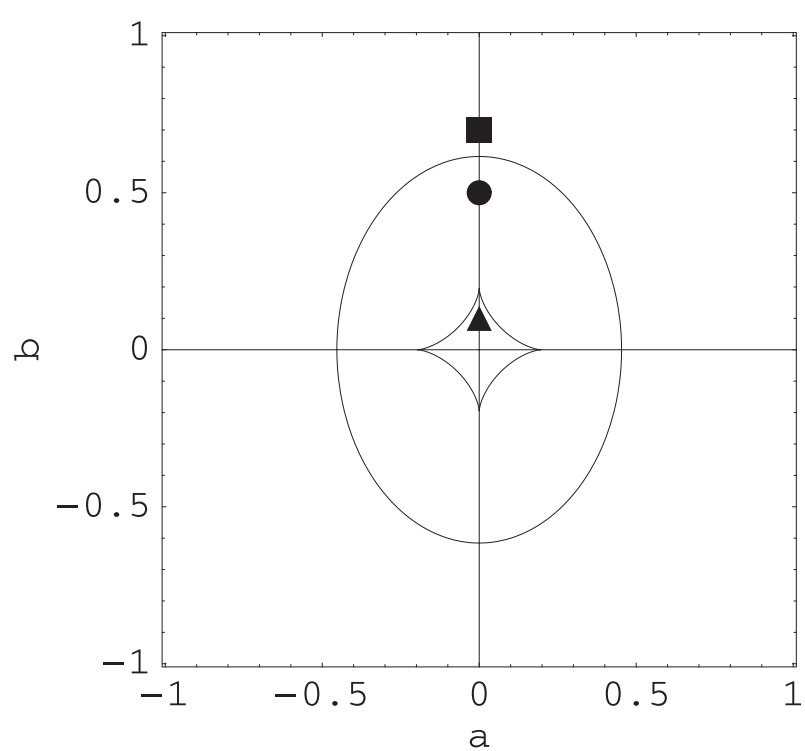

Fig. 1. Caustics for a cored isothermal ellipsoidal lens for $e=0.1$ and $c=0.2$. Sources are located at $(0,0.1),(0,0.5)$ and $(0,0.7)$, denoted by the triangle, filled disk and square, respectively.

with respect to $r$ to obtain a $\phi$-parameter representation. This $r$-representation becomes

$(x, y)=\left( \pm r \sqrt{\frac{1+h}{2}}, \pm r \sqrt{\frac{1-h}{2}}\right)$

where we defined

$$
\begin{aligned}
h= & (e R)^{-1}\left(\left(R+c^{2}\right)^{3 / 2}-\left(R+2 c^{2}\right)\right. \\
& \left.+c^{2}\left(1-e^{2}\right)\left(R+c^{2}\right)^{-1 / 2}\right) .
\end{aligned}
$$

The allowed regions for $r=\sqrt{R}$ are given by Eqs. (25)-(30) depending on $c$ and $e$. Substituting this representation into Eqs. (3) and (4), we obtain a representation of the caustics as

$a(r)= \pm r\left(1-\frac{1-e}{\sqrt{r^{2}+c^{2}}}\right) \sqrt{\frac{1+h}{2}}$
$b(r)= \pm r\left(1-\frac{1+e}{\sqrt{r^{2}+c^{2}}}\right) \sqrt{\frac{1-h}{2}}$

As an illustration, the caustics and critical curves are shown for $e=0.1$ and $c=0.2$ in Figs. 1 and 2, respectively. We can see two curves in both figures as discussed above. The inner caustics in Fig. 1 look like an asteroid. However, it is not exact but approximate, since Eqs. (33) and (34) are not the equation for asteroids (Asada et al. 2003).

\section{Conclusion}

We have studied the effect of the core radius of an isothermal ellipsoid on the caustics and critical curves. For this purpose, we have obtained a simple parametric representation of the critical curves. The caustics have been expressed also with the same parameter. It turns out that a nonvanishing core radius changes the shape and number of the caustics and critical curves. These 


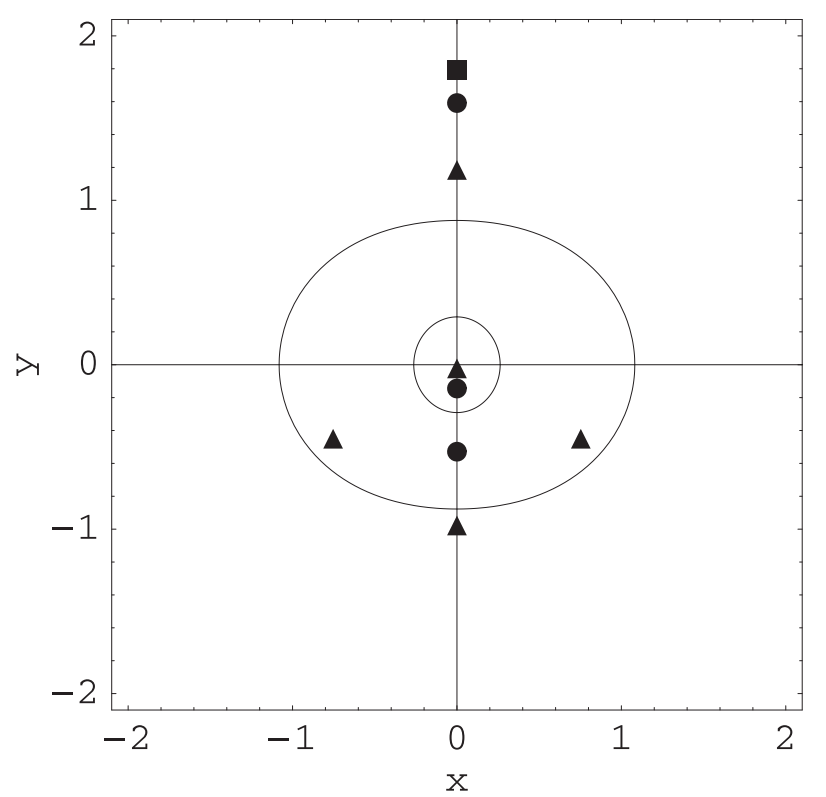

Fig. 2. Critical curves for a cored isothermal ellipsoidal lens for $e=$ 0.1 and $c=0.2$. The images correspond to the sources in Fig. 1 .

curves are two for a small core radius $c<1-e$, one for a quite large case $1-e<c<1+e$, and disappear for an extremely large one $1+e<c$, where $c$ is the angular core radius and $e$ is the ellipticity of the isothermal ellipsoid. However, the latter two cases are not realistic in astronomical situations as discussed above.

We must perform numerical computations for various values of the parameters in order to obtain the caustics and critical curves. The present expression is analytic. Hence, it enables us to save time and reach more accurate results, for instance in rapid and accurate estimations of a lensing event rate, fittings to observational data, etc.

Acknowledgements. The author would like to thank M. Bartelmann, Y. Mellier and M. Kasai for useful conversations. He would like to thank L. Blanchet for hospitality at the Institute for Astrophysics at Paris. This work was supported by a fellowship for visiting scholars from the Ministry of Education of Japan.

\section{References}

Asada, H. 2002, A\&A, 390, L11

Asada, H., Kasai T., \& Kasai M. 2002, Prog. Theor. Phys., 108, 1031 Asada, H., Hamana T., \& Kasai M. 2003, A\&A, 397, 825

Binney, J., \& Tremaine, S. 1987, Galactic Dynamics (Princeton University Press)

Schneider, P., Ehlers, J., \& Falco, E. E. 1992, Gravitational Lenses (Springer-Verlag)

van der Waerden B. L. 1966, Algebra I (Springer)

Wolfram, S. 2000, The Mathematica Book, 4th ed. (Cambridge: Cambridge University Press) 